\title{
A Multidisciplinary Approach for the Management of Extremely Abraded Dentition
}

\section{ISSN: 2637-7764}

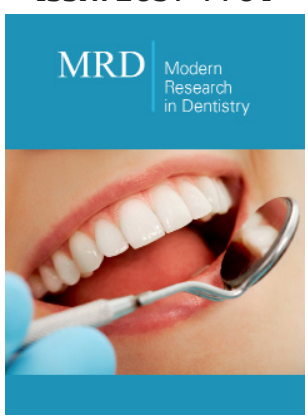

*Corresponding author: Dr.Tzanakakis Emmanouil-Georgios, Department of Operative Dentistry, Dental School of Athens, National and Kapodistrian University of Athens, Greece

Submission: 非June 25, 2020

Published: 佔July 22, 2020

Volume 5 - Issue 3

How to cite this article: Dr.Tzanakakis Emmanouil-Georgios. A Multidisciplinary Approach for the Management of Extremely Abraded Dentition. Mod Res Dent. 5(3). MRD. 000612. 2020.

DOI: $10.31031 /$ MRD.2020.05.000612

Copyright@ Dr.Tzanakakis Emmanouil-Georgios, This article is distributed under the terms of the Creative Commons Attribution 4.0 International License, which permits unrestricted use and redistribution provided that the original author and source are credited.

\section{Dr:Tzanakakis Emmanouil-Georgios*}

Department of Operative Dentistry, Dental School of Athens, National and Kapodistrian University of Athens, Greece

\section{Summary}

Restoring extremely abraded dentition is considered as one of the most demanding and time consuming clinical challenges. Patients are often unaware of the complexity of these treatment plans and are usually reluctant to accept major prosthetic interventions. Proper coordination of the clinicians involved in the treatment plan is essential and saves valuable clinical time. This clinical report describes thoroughly a complicated treatment plan that involves periodontic and endodontic treatment, dental implants, orthodontic extrusion and a full-mouth rehabilitation with a combination of all-ceramic and metal-ceramic restorations. In this case report the role of temporary restorations in increased VDO is emphasized and with an extended discussion on clinical steps.

Keywords: Abraded dentition; VDO; Bruxism; Metal-ceramic; All-ceramic

Abbreviations: TMJ: Temporomandibular Joint; VDO: Vertical Dimension of Occlusion; RPD: Removable Partial Denture

\section{Introduction}

The management of complicated cases of extremely abraded dentition is a difficult task for the clinician. It has a negative impact on quality of life comparable with that of edentulousness [1]. The reconstruction of dental arches requires extensive restorative treatment [2,3]. Every patient requires unique treatment, however most of them at the beginning of treatment are not aware of the cost and time that is necessary for a proper and long term treatment plan and postpone the therapeutic interventions. Moreover they are usually confused because specialized clinicians may propose different treatment plans. A team of experienced clinicians can suggest an appropriate treatment planning [2]. Nowadays, the increase of mechanical strength and adhesive potential of new dental materials may offer new possibilities in reconstructive techniques and digital workflow can minimize invasive tooth preparations $[4,5]$. Bruxism is a repetitive parafunctional activity and the main cause for temporomandibular disorders (TMD) [6]. Wear facets along with masseter muscle hypertrophy other clinical are common findings that indicate the presence of bruxism [6]. Masticatory forces in bruxing patients are much higher than maximum biting forces measured during chewing cycles, which partially explains the catastrophic impact to dental restorative materials. It is widely considered that bruxism has also a negative impact on the periodontal tissues and is a main cause of loss of osseointegration of dental implants [7-9]. Loss of posterior support causes difficulty in mastication especially in young and middle aged population. In these patients, anterior teeth are overloaded. The results are either tooth mobility or extensive wear of the clinical crown. Restoration of posterior support is necessary as early as possible with provisional restorations to restore periodontic and mechanical overload of the remaining dentition [2].

Fixed implants restorations are more attractive for the patients especially when the alternative treatment plan includes a removable device. If a patient can afford the increased cost and accepts the minor surgical procedure, implants are a reliable solution to partial edentulism [10]. The aim of this case report was to analyze the therapeutic management of a patient presenting generalized excessive tooth wear in the mandibular dentition involving 
decreased VDO and loss of posterior support. The treatment included a combination of periodontic and endodontic therapies, implants placement, minor orthodontic treatment and optimum prosthodontic rehabilitation with fixed dental prostheses, cast posts and all ceramic veneers in increased VDO.

\section{Case Report}

\section{Patient presentation}

The Caucasian 53-year-old male patient was seeking treatment. His chief complaint was difficulty in chewing and his anticipation for a fixed restoration (Figure 1).

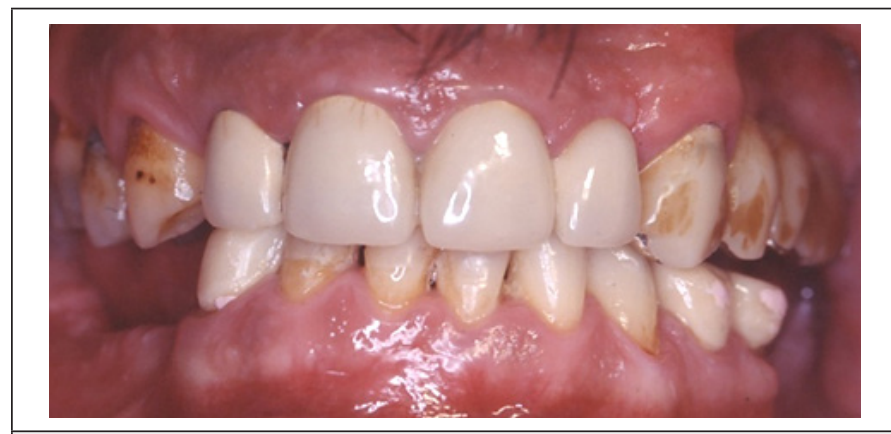

Figure 1: Preoperative intraoral frontal view.

\section{Initial clinical situation, radiographic examination}

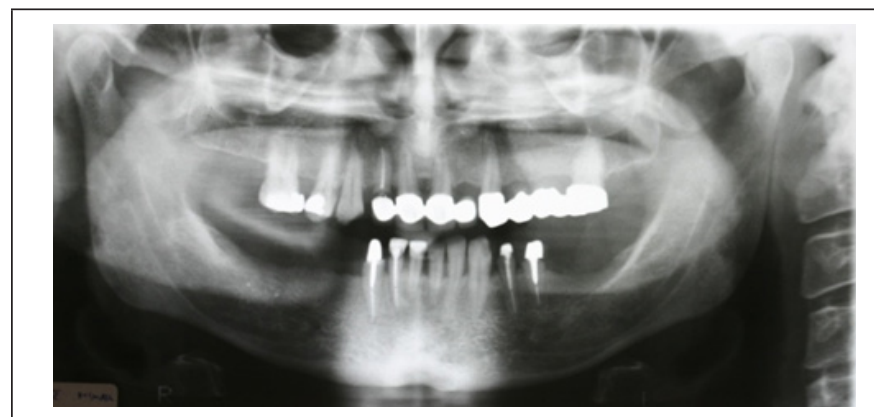

Figure 2: Preoperative panoramic radiograph.

The patient reported a free medical history, but admitted smoking habit (20+cig/day). He was not taking any medication and reported pain in facial muscles during stressful periods. The patient was subjected to thorough clinical and radiographic examination (panoramic x-ray) (Figure 2). Intraorally, he presented severe abrasion most observed in mandibulary dentition, reduced VDO, and several missing mandibular teeth (\#44,45,46,47 and 36,37). In the maxilla, a 4-unit metal ceramic bridge with a cantilever was found on the incisors $\{12-11-21-(22)\}$, and one 4-unit metal-acrylic bridge in the posterior segment $\{23-(24)-(26)-27\}$. The visible diastemmas between \#12,\#13 and \#13,\#14 were due to a missing premolar \#15 which probably caused distal migration of \#14 and 13 (Figure 3-5). The smile line was evaluated as medium (Figure 6). Secondary caries was visible in amalgam restorations in \#14 and \#16 and was inspected under the abutment \#12. A significant change in the occlusal plane was obvious, defined by significant protrusion of \#13, \#14 and \#16. In the mandible acrylic crowns were positioned in \#44,33, 34. Initial radiographic examination revealed endodontic therapies in \#12, 34, 35, 42 and \#43 and it was estimated that the bone level averaged at 70\%. Clinical examination of the stomatognathic system revealed bilateral myalgia of the lateral pterygoid muscles on palpation, unilateral clicking on the left TMJ and limitation in mouth opening. All other masseter muscles were free of symptoms.
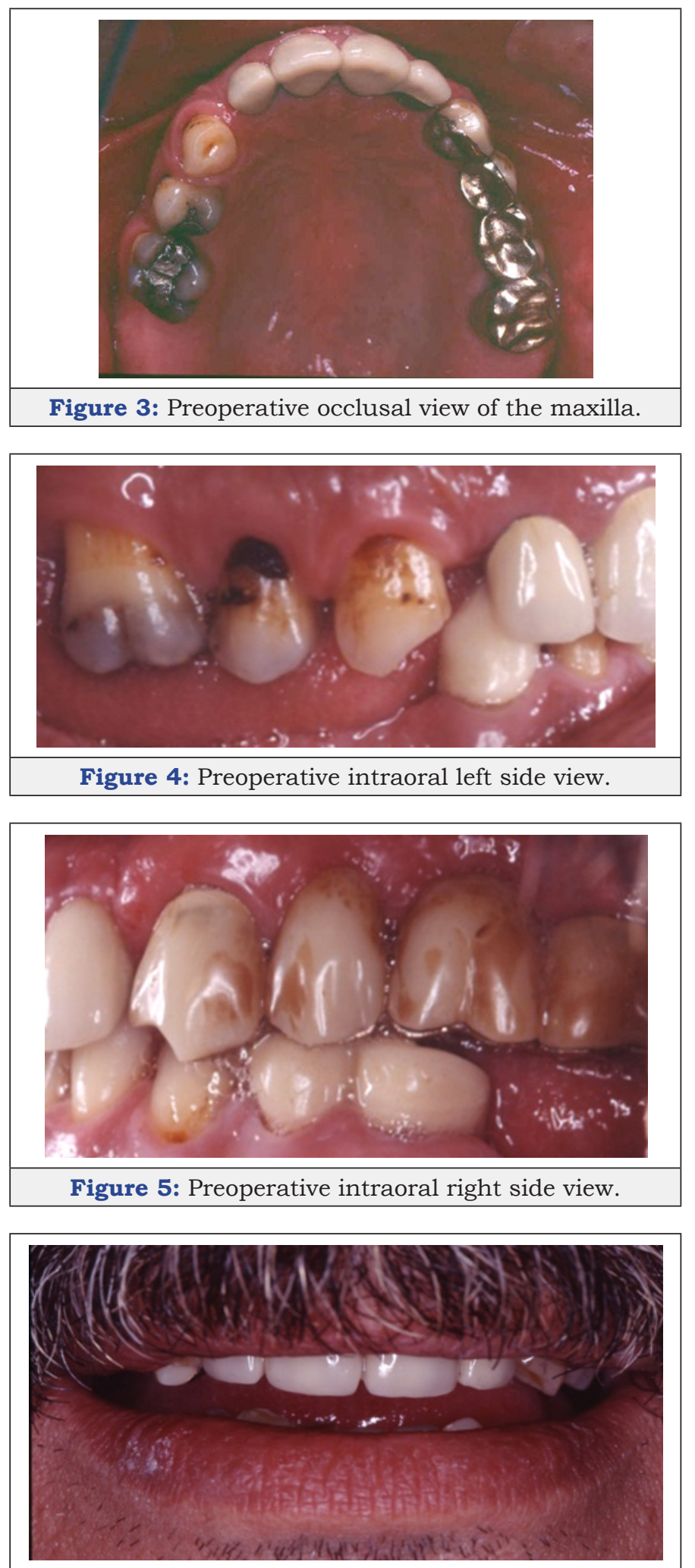

Figure 6: Extraoral frontal view showing a medium smile line. 


\section{Preparatory clinical steps}

Initial alginate impressions were taken for the fabrication of initial study casts. Prior to jaw registrations an initial occlusal splint was fabricated and given to the patient in order to reduce TMJ symptoms (Figure 7). Using a transparent $2 \mathrm{~mm}$ hard plate after thermal processing and vacuum on maxillary study cast a retentive basis was fabricated. The splint was fabricated chairside by adding autocuring acrylic resin (Jet, Lang, USA) and after occlusal reduction the increase of VDO was set to $5 \mathrm{~mm}$. Two weeks later a face bow registration (Hanau) and three centric relation registrations were taken to ensure the accuracy of the procedure. (Figure 8). Study casts were mounted in a semi adjustable articulator (Hanau H2). The static occlusion analysis revealed increased vertical overlap, and a discrepancy of occlusal plane in quadrant \#1 (Figure 9).
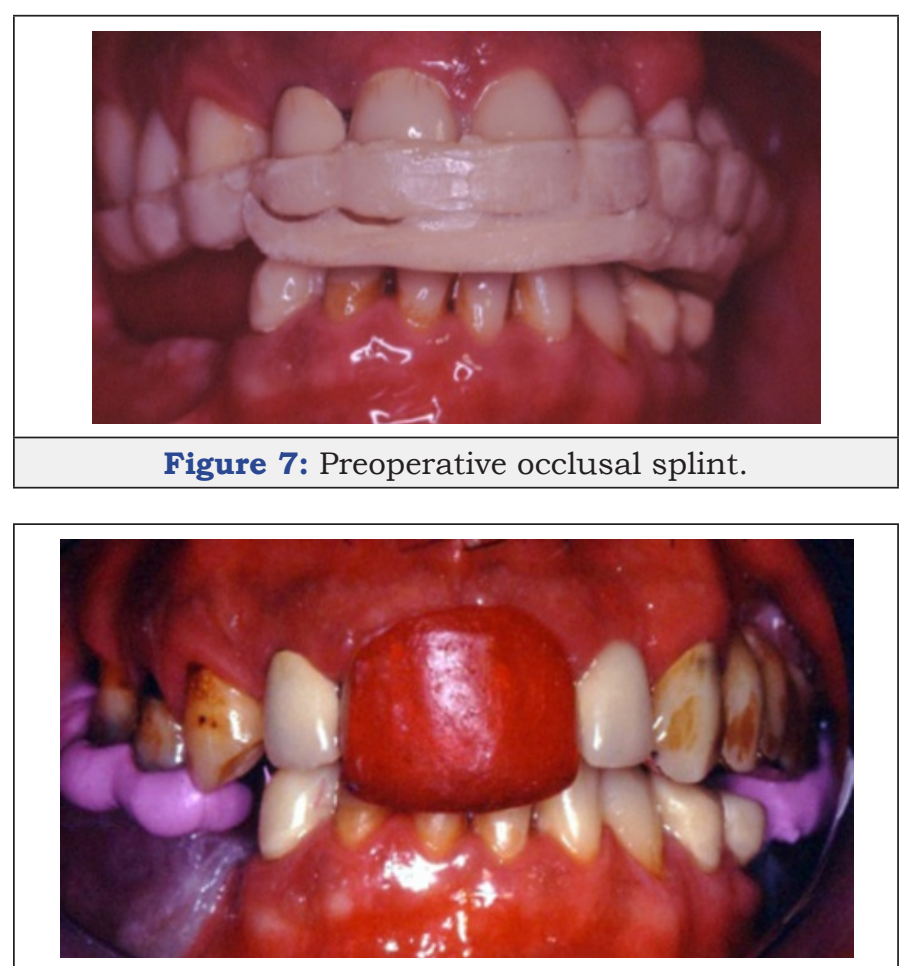

Figure 8: Initial centric relation registration(anterior deprogrammer technique).

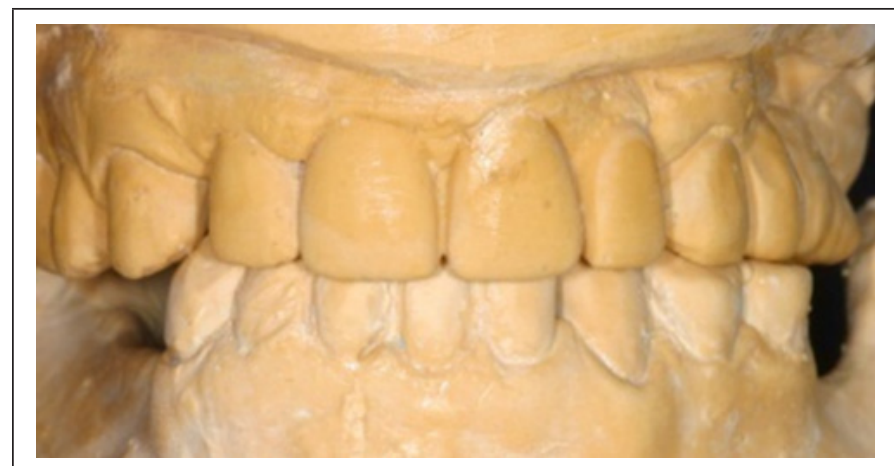

Figure 9: Study casts (frontal view).

The diagnostic wax-up was performed in an increased vertical dimension of occlusion (approximately by $3 \mathrm{~mm}$ ). The maxillary teeth were imposed in minimal modifications; however the diastemma between \#13 and 15 was difficult to be eliminated. The increase in VDO was mainly attributed to the mandibular teeth in order to alter frontal abraded dentition and to create the important prosthetic space for the posterior missing teeth. The existent prosthetic restorations in the maxilla were slightly differentiated in the wax up, however significant occlusal reduction was performed in teeth \#15 and \#16 (Figure 4 \& 10). These modifications corrected occlusal plane.

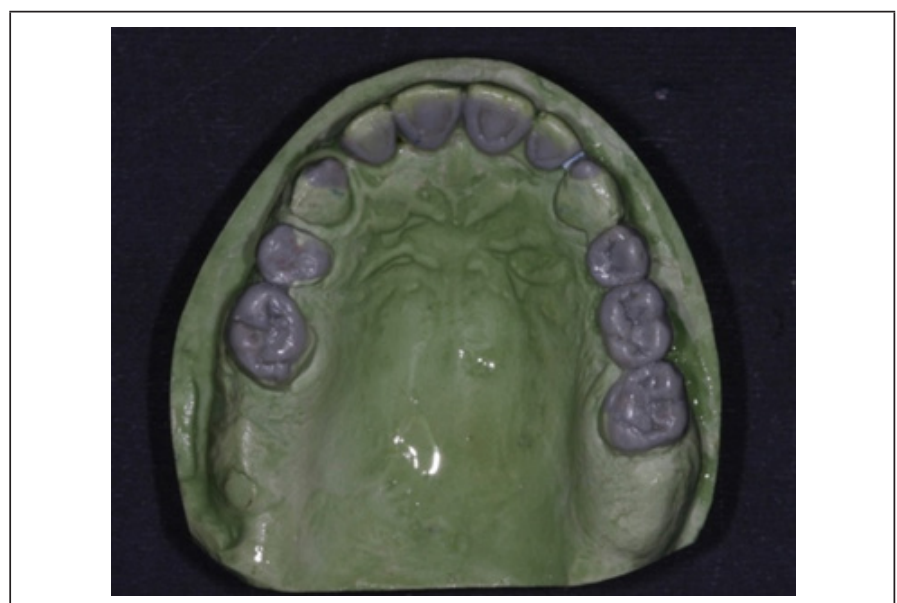

Figure 10a: Diagnostic wax up occlusal view of the maxilla.
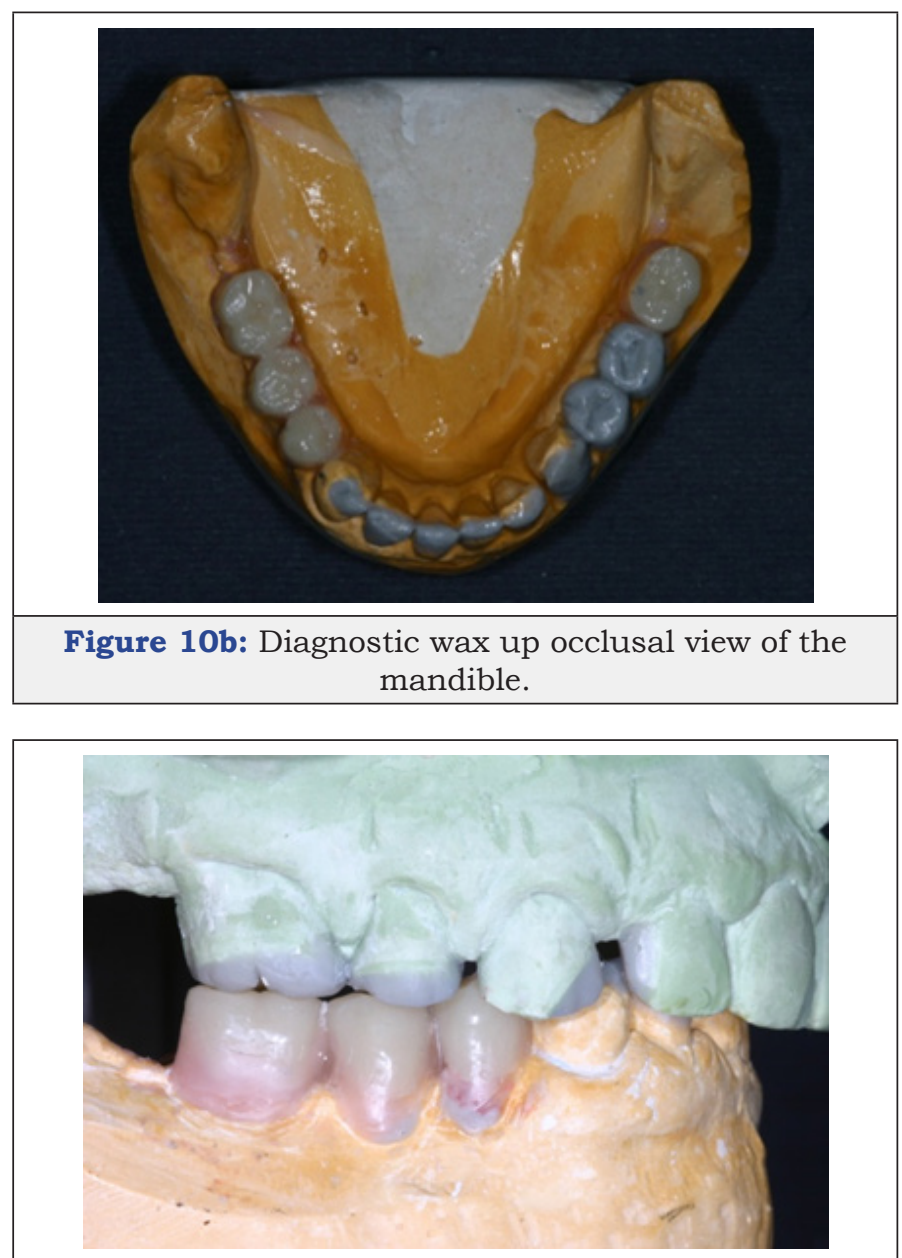

Figure 10c: Right side view of the diagnostic wax up. 


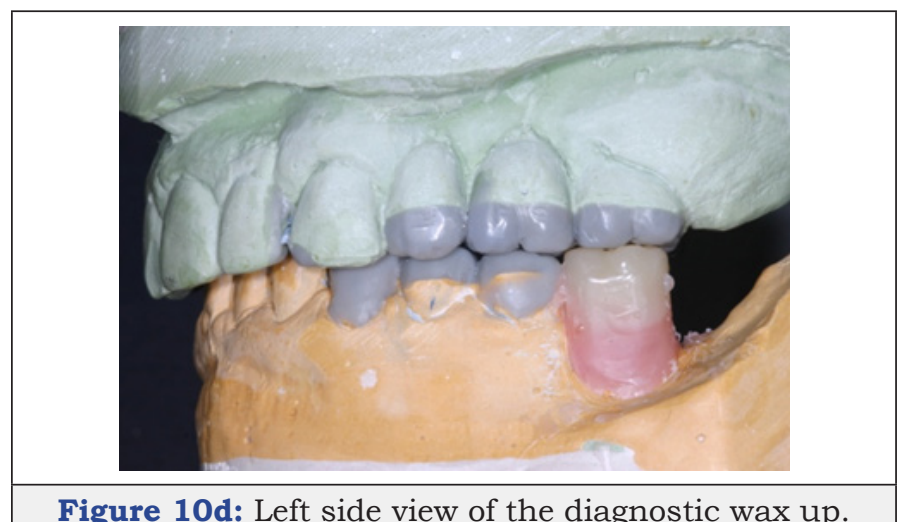

Figure 10d: Left side view of the diagnostic wax up.

\section{Initial treatment plan}

The initial treatment plan was maxillary and mandibulary fullarch reconstructions that included all necessary clinical steps:

1. Periodontal treatment,

2. provisional restorations in existing VDO,

3. provisional restorations in increased VDO,

4. Endodontic treatment of \#12,15,16,

5. surgical periodontal treatment and implant placement,

6. final restorations with metal-ceramic bridges in the maxilla and implant FDPs and

7. a stabilization splint.

\section{Clinical steps}

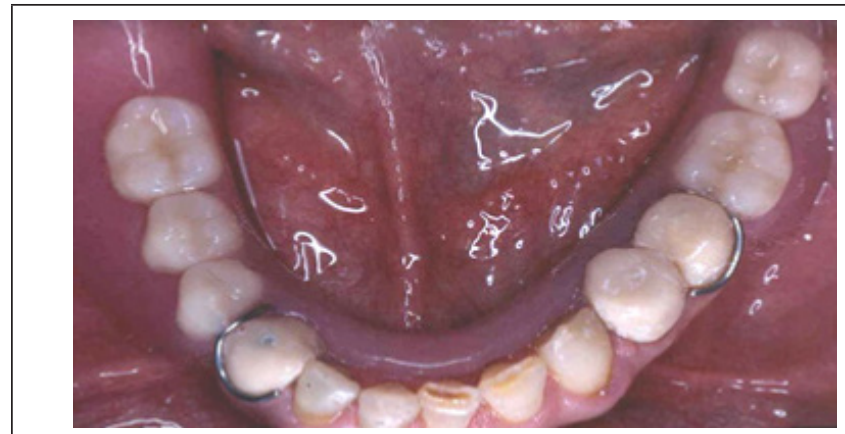

Figure 11: Temporary mandibular removable partial denture.

Clinical periodontal examination revealed generalized moderate chronic periodontitis. Upon crown removal secondary caries was found on most abutments. Temporary acrylic bridges shaped as the existing restorations without change in VDO were fabricated after thorough caries removal and minor resin restoration in selected abutments. In the next appointment a laboratory heat-cured acrylic restoration was inserted in increased VDO in shape corresponding to the diagnostic wax-up. In the meantime acrylic crowns were fabricated in the mandible combined with a temporary acrylic RPD similar to previous wax-up (Figure 12). At the the first recall a cracking was noticed in the maxillary acrylic restoration and it was decided to reinforce the acrylic with a "rochette type design" metal device (Figure 11). The patient followed a recall program for the next 3 months to adopt the new increased VDO. The patient was subjected to phase I periodontal treatment, which included oral hygiene instructions, patient motivation, supragingival debridement and root scaling in four quadrants and revaluation of the periodontal tissues at six weeks. All endodontic therapies and cast post restorations were completed during this period. Meanwhile surgical procedures and implant placement were scheduled after the periodontal revaluation.
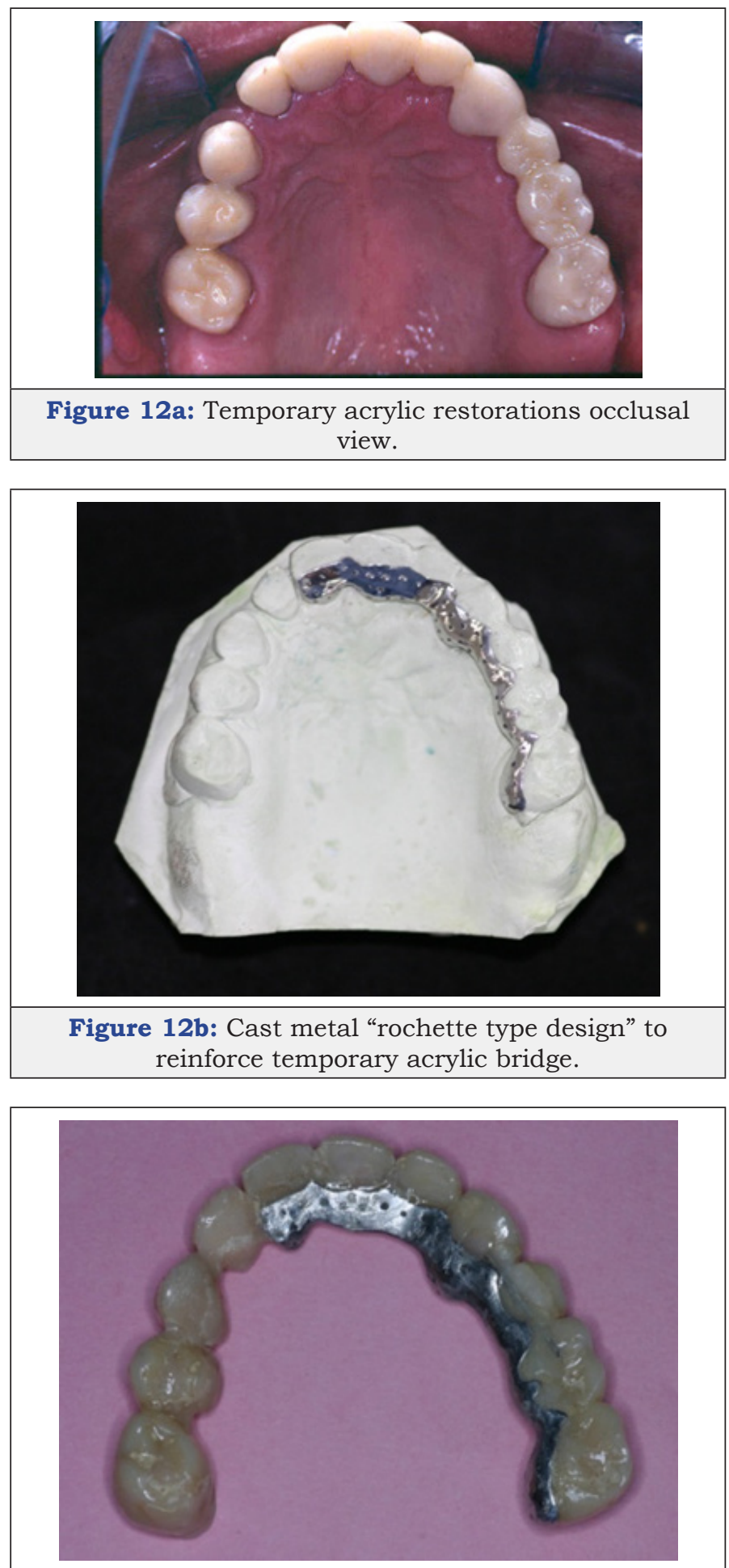

Figure 12c: Reinforced hybrid temporary acrylic restoration after 3 months of intraoral service. 


\section{Final treatment plan}

Revaluation, at six weeks, revealed that the inflammation of the periodontal tissues was significantly reduced and periodontal surgery for pocket elimination was not required. However teeth \#35 and 12 necessitated surgical crown lengthening to preserve the biological width. Implant placement in \#36 was designed with simultaneous crown exposure of \#35 in order to shorten treatment plan time. An alternative procedure for crown lengthening was proposed for \#12, thus an orthodontic extrusion was implemented. Orthodontic therapy required the separation of acrylic crown \#12 from the splinted frontal bridge and the position of metal brackets in adjacent teeth (Figure 13). In less than three months, a significant extrusion of abutment \#12 was completed (Figure $14 \& 15$ ). In order to stabilize the position of the extruded \#12, the acrylic crown was splinted with adjacent acrylic ones. Surgical placement of implant \#36 was combined with surgical exposure of \#35. Three implants in positions \#44, 45, 46 were also inserted.
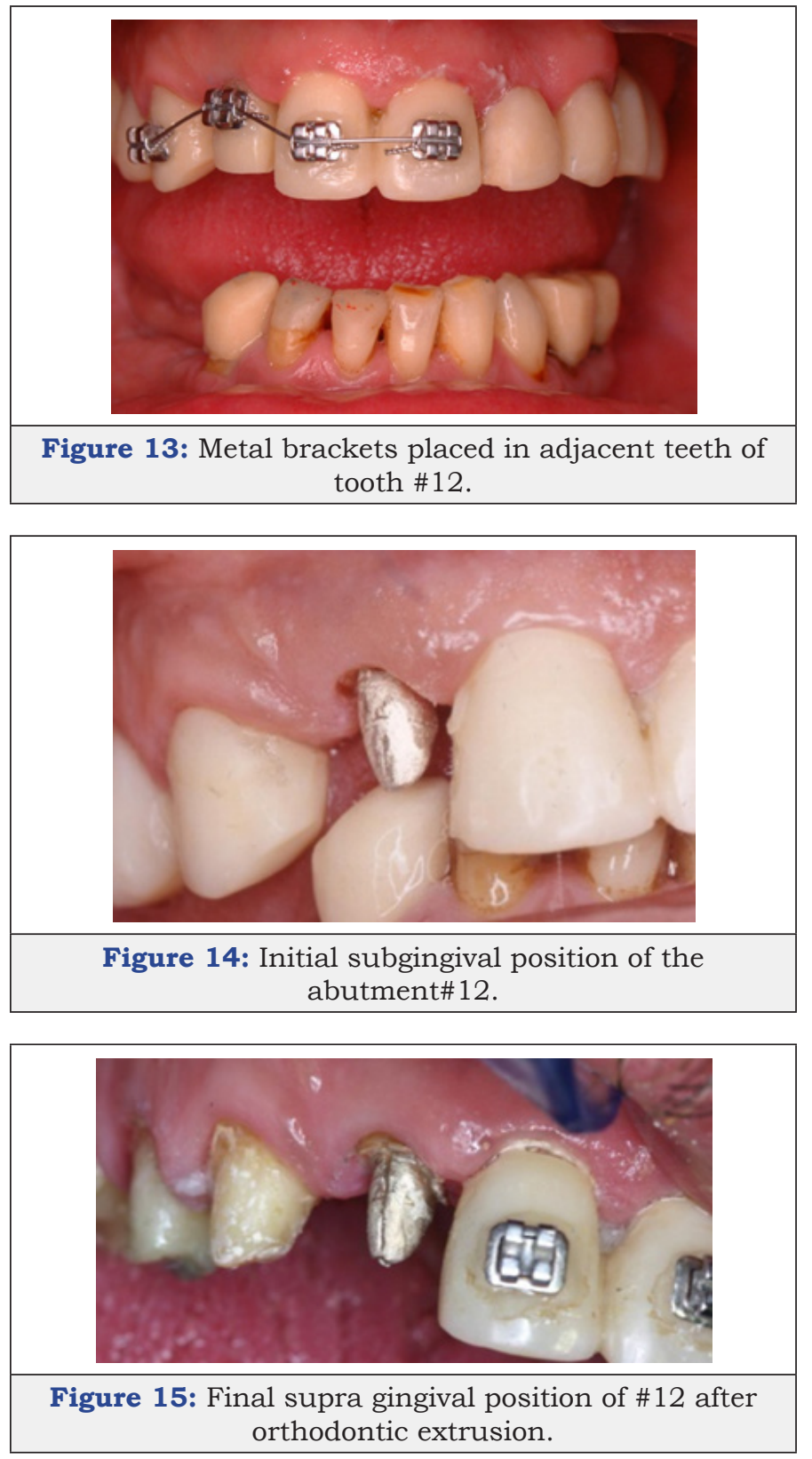

\section{Final restorations}

The final preparations were observed in two study casts to improve the parallelism and ensure accurate path of the insertion for the final prostheses. Final preparations and relining of the provisional restorations followed. Final impressions were performed using polyvinyl-siloxane (Aquasil, Dentsply, Germany) with the two-step double mixing technique (stock tray, double cord technique) in the maxilla. Mandibular impression was performed with an open tray technique combining implant and teeth abutments with polyether material (Impregum, 3M, USA). In next appointment the accuracy of final casts was examined and jaw relation recordings were performed. The position of maxillary cast was transferred with a Whip-Mix face bow and centric relation (CR) was registered with a custom made acrylic Lucia jig (anterior deprogrammer) and an implant retained base plate in the mandible to ensure accurate registration. Dental and implant abutments metal try-in were completed in two steps (Figure 16). After thorough verification of accuracy of fit and jaw registrations, ceramic veneering was checked for proper occlusion (Figure 17,18). Finally all restorations were cemented with adhesive resin cement (Panavia F2.0, Kuraray, Japan), except for the implant restorations which were cemented with a provisional cement (Temp-bond, Kerr, Germany).

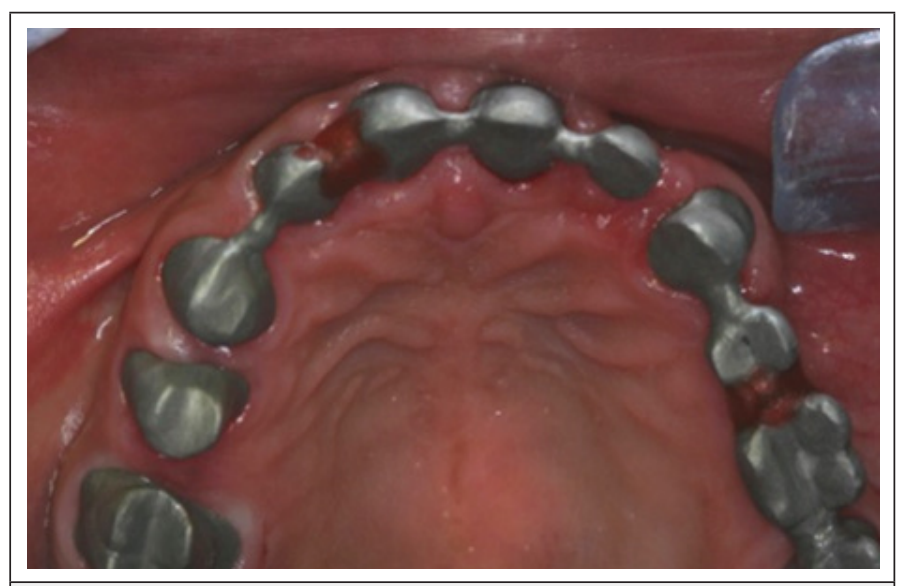

Figure 16: Dental abutments metal try in the maxilla.

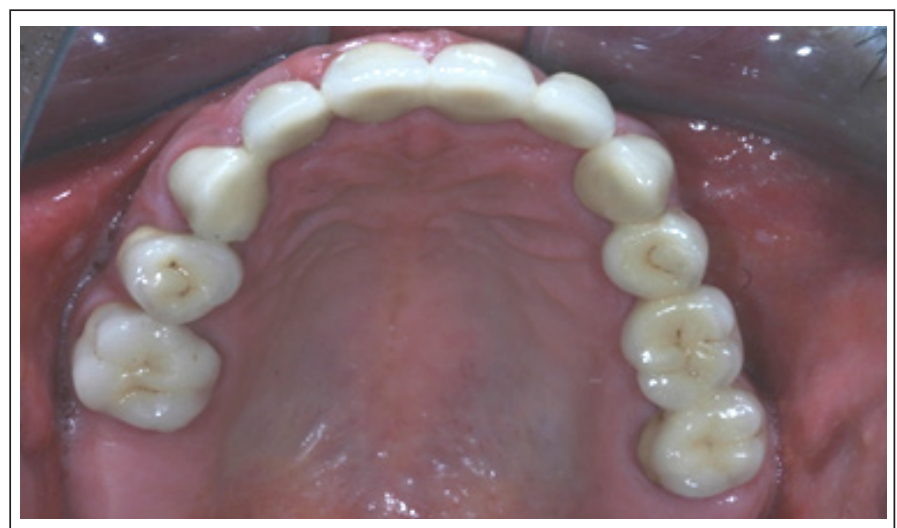

Figure 17: Final restorations occlusal view of the maxilla. 


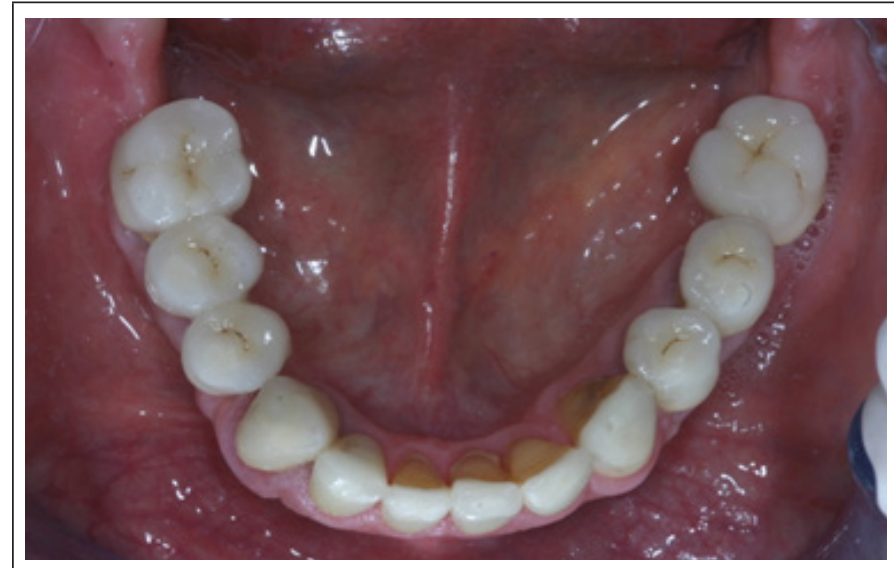

Figure 18: Final restorations occlusal view of the mandible.

\section{Preparations for veneers, final result}

In order to optimize final aesthetics the 5 mandibular anteriors were prepared for all ceramic restorations. Four all ceramic veneers (E-max Ivoclar Co, Liechtenstein) and an all ceramic crown (\#42) completed the final restorations. A final occlusal splint was also fabricated chairside (prior to the final cementation of ceramic veneers) for the protection of final restoration and control of TMJ function (Figure 19,20). Minimal occlusal modifications of the splint were necessary after final cementation of mandibular anteriors. The final radiographic examination was kept as a record for future re-examinations (Figure 21). Finally, the patient was enrolled to a 3 months recall program. In each recall and maintenance visit, the periodontal tissues, the abutments and the stomatognathic system were thoroughly examined, preventive regimens were applied and patient compliance was assessed. Two years later, the excellent periodontal condition and performance of the restoration was registrated (Figure 22-24). The patient complied with proper oral hygiene, reduced smoking and adopted a regular use of the occlusal splint. Strict recall and maintenance visits were necessary for long term success of this full mouth rehabilitation. The patient stopped smoking immediately after 6 months and changed nutritional habits in order to eliminate secondary caries.

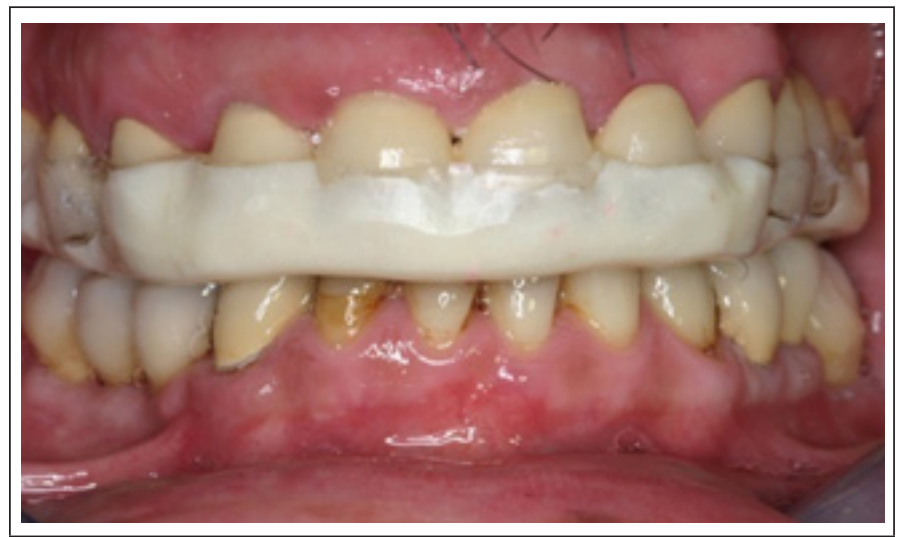

Figure 19: Occlusal splint for the protection of final restoration.

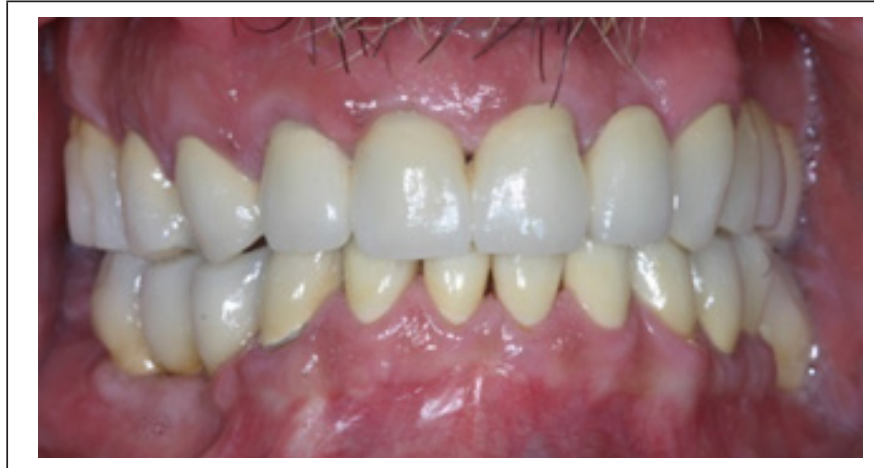

Figure 20: Final restorations after cementation of the all ceramic veneers.
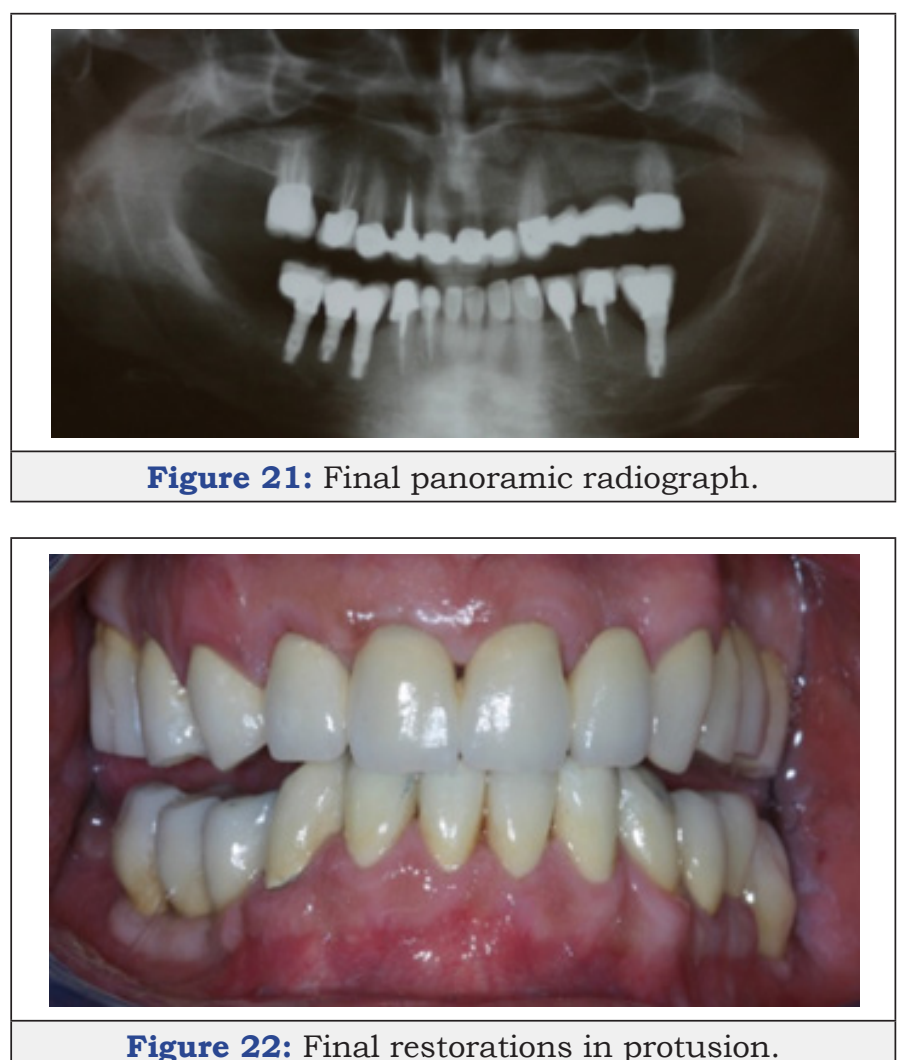

Figure 22: Final restorations in protusion.

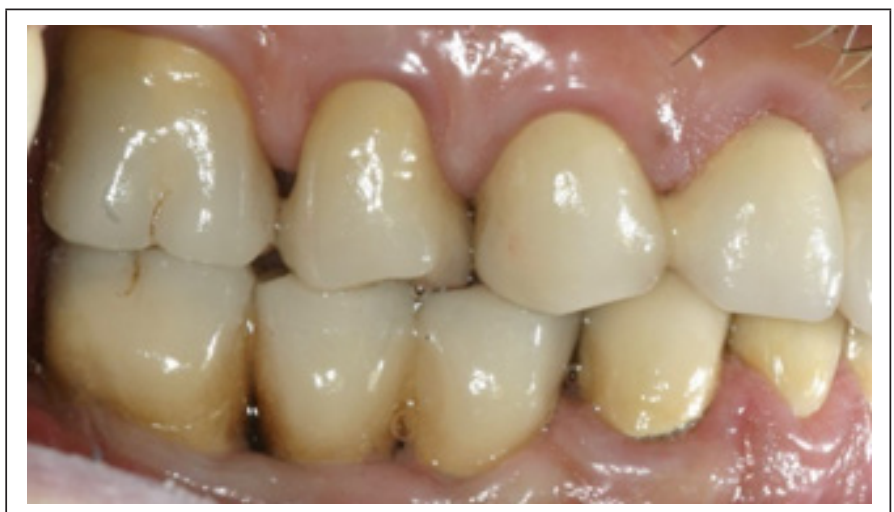

Figure 23: Right side view of final restorations (one year recall). 


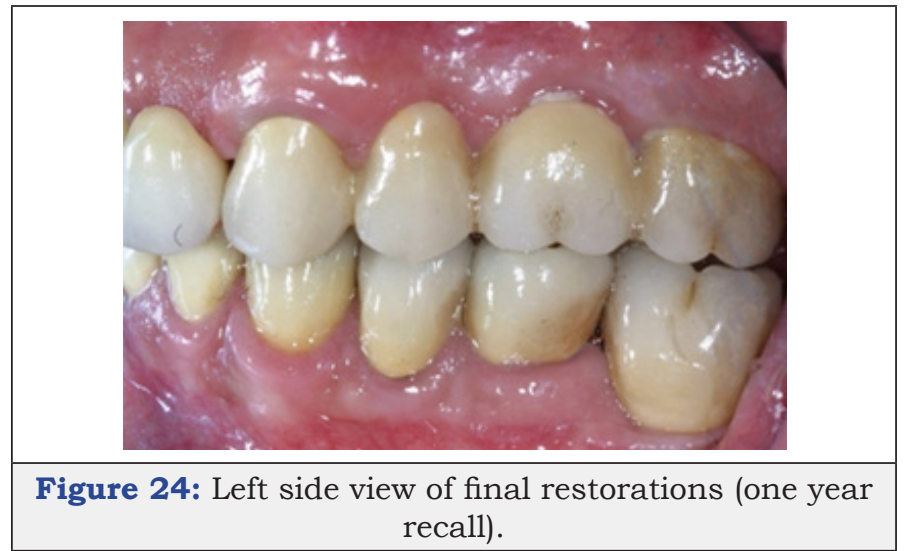

\section{Discussion}

This report illustrates a multidisciplinary approach to reconstruction of a complex prosthodontic case in increased vertical dimension of occlusion. Four post graduate students were involved to achieve a final result. The aim of all clinicians was a pleasant outcome fulfilling the desire of the patient. The development of excellent communication between clinicians and the patient was crucial especially in this prolonged time of dental therapies. All treatment plans were discussed with the patient and diagnostic wax up outlined the final result. Provisional acrylic restorations ensured a gradual adaptation of the patient in increased VDO. Stability and reinforcement of provisional restorations facilitated periodontic, endodontic and orthodontic interventions. Sometimes the increase of VDO is inevitable in order to achieve proper function and better esthetics. Arbitrary increase of VDO should be accomplished after prolonged trial period [2]. Moreover the increase of VDO created adequate intralveolar space for implant placement in segments \#3 and \#4. Reconstruction ceramic materials require sufficient restorative space for better esthetics.

An anterior metal-ceramic bridge and a posterior metalacrylic restoration were in function in the maxilla for more than a decade in this patient without failing outcome. In order to improve esthetics with a predictable prognosis a combination of metalceramic and all-ceramic restorations were selected in this case, since they seem to be a safe choice in cases of high load conditions [11]. Restorative procedures are differentiated in patients with bruxism. Maximum retention is required from dental abutments and adhesive cementation techniques optimize long term stability of restorations in bruxists [12]. In the maxilla and in the mandible in posterior quadrants metal ceramic bridges and crowns were fabricated. Implant cement retained crowns \#44, 45, 46 were splinted for maximum retention. All ceramic crown and veneers were adhesive cemented following $\mathrm{HF}$ and silanization protocol [13]. Single tooth orthodontic extrusion as alternative to crown lengthening technique is a safe method of treating teeth with subgingival finish line. Biologic width can be reserved without soft and hard tissue removal [14]. Orthodontic extrusion in \#12 eliminated surgical demands in anterior segment in the maxilla $[15,16]$. This method is considered as an adjunct to periodontal therapy and restores the biological width without sacrifice of bone structure $[14,17]$. Implant fixed restorations in patients with mandibular class II edentulism present an excellent alternative to a RPD since the adoption to a removable prosthesis is often difficult. In this case report the provisional mucosal supported mandibular RPD revealed the intensity of this problem to this patient, even though a final tooth-mucosa borne RPD might present less discomfort [18-20].

\section{Alternative treatment plans}

A. In the maxilla 2 implants in \#24 and 25 would eliminate the necessity for the bridge \#23-(24)-(25)-26. However, the abutments in \#23 and 26 were already prepared for full coverage metal-acrylic restorations and required minimal additional preparation for the fabrication of a new metal ceramic bridge. The cost/benefit ratio from implant placement was not considered important.

B. In the mandible the \#4 quadrant an alternative option for treatment would be the placement of two implants in \#44 and \#46 positions and the construction of a metal ceramic 3-unit bridge. However, the selection of 3 implants was considered safer due to bruxism (REF).

C. In the mandible conventional prosthodontics with metal ceramic crowns and a class I Kennedy RPD was the second alternative treatment plan. However, the patient was not satisfied with the provisional acrylic RPD and preferred a fixed restoration. The conventional restoration would lower the total cost and time of dental therapy.

\section{Conclusion}

Conventional fixed prosthodontics combined with implant restorations, are a challenge for the restoration of severely abraded dentition. Orthodontic extrusion is an excellent alternative to surgical lengthening in the anteriors. Proper design of the treatment plan, a strict recall system and maintenance visits minimize the incidence of clinical complications.

\section{Acknowledgement}

The author would like to thank Assoc.Prof. Kourtis S. and Assistant Prof.Roussou I. from the department of Prosthodontics for their help.

\section{References}

1. Papagianni CE, Van der Meulen MJ, Naeije M, Lobbezoo F (2013) Oral health-related quality of life in patients with tooth wear. J Oral Rehabil. 40(3): 185-190.

2. Turner KA, Missirlian DM (1984) Restoration of the extremely worn dentition. J Prosthet Dent 52(4): 467-474.

3. Stewart B (1998) Restoration of the severely worn dentition using a systematized approach for a predictable prognosis. Int J Periodontics Rest Dent 18(1): 46-57.

4. Loomans B, Opdam N, Attin T, Bartlett D, Edelhoff D, et al. (2017) Severe tooth wear: European consensus statement on management guidelines. J Adhes Dent 19(2): 111-119.

5. Lee JH, Kim SH, Han JS, Yeo IL, Yoon HI (2019) Contemporary fullmouth rehabilitation using a digital smile design in combination with 
conventional and computer-aided design/manufacturing restorative materials in a patient with bruxism: A case report. Medicine 98(48): e18164.

6. Lobbezoo F, Ahlberg J, Raphael KG, Wetselaar P, Glaros AG, et al. (2018) International consensus on the assessment of bruxism: Report of a work in progress. J Oral Rehabil 45(11): 837-844.

7. Jacobs R, De Laat A (2000) Bruxism and overload periodontium and implants. Ned Tijdschr Tandheelkd 107(7): 281-284.

8. McCoy G (2002) Recognizing and managing parafunction in the reconstruction and maintenance of the oral implant patient. Implant Dent 11(1): 19-27.

9. Lobezoo F, Browers JEIG, Cune MS, Naeije M (2006) Dental implants in patients with bruxing habits. J Oral Rehabil 33(2): 152-159.

10. Lindquist LW, Carlsson GE, Jemt T (1996) A prospective 15-year followup study of mandibular fixed prostheses supported by osseointegrated implants. Clin Oral Implant Res. 7(4): 329-336.

11. Dahl BL, Carlsson GE, Ekfeldt A (1993) Occlusal wear of teeth and restorative materials. A review of classification, etiology, mechanisms of wear, and some aspects of restorative procedures. Acta Odontol Scand 51(5): 299-311.

12. Misch CE (2002) The effect of bruxism on treatment planning for dental implants. Dent Today 21(9): 76-81.
13. Hill E, Lott J (2011) A clinically focused discussion of luting materials. Australian Dent J 56(suppl 1): 67-76.

14. Minsk LQ (2000) Orthodontic tooth extrusion as an adjunct to periodontal therapy. Compendium 21(9): 768-775.

15. Ingber JS (1976) Forced eruption: Part II. A method of treating non restorable teeth-periodontal and restorative considerations. J Periodontol 47(4): 203-216.

16. Ivey D, Calhoun R, Kemp W, Dorfman HS, Wheless JE (1980) Orthodontic extrusion: Its use in restorative dentistry. J Prosthet Dent 43(4): 401407.

17. Pontoniero R, Celenza F, Ricci G (1987) Rapid extrusion with fiber resection: A combined orthodontic-periodontic treatment modality. Int J Periodont Res Dent 7(5): 30-43.

18. Giffin KM (1996) Solving the distal extension removable partial denture base movement dilemma: a clinical report. J Prosthet Dent 76: 347-349.

19. Belser UC, Mericske Stern R, Bernard JP, Taylor TD (2000) Prosthetic management of the partially dentate patient with fixed implant restorations. Clinical Oral Implants Research 11(suppl 1): 126-145.

20. Thymi M, Verhoeff MC, Visscher CM, Lobbezoo F (2020) Patient-based experiences with the use of an ambulatory electromyographic device for the assessment of masticatory muscle activity during sleep. J Oral Rehabil 47(5): 557-566. 